\title{
Operations Scheduling of Sugarcane Production Using Classical GERT Method (Part I: Land Preparation, Planting and Preserve Operations)
}

\author{
Nasim Monjezi (Corresponding author) \\ Department of Agricultural Machinery, Faculty of Agriculture \\ Shahid Chamran University, PO box 6166616453, Ahvaz, Iran \\ Tel: 0098-930-201-6425Ｅ-mail: N-monjezi@phdstu.scu.ac.ir
}

Mohammad Javad Sheikhdavoodi

Department of Agricultural Machinery, Faculty of Agriculture

Shahid Chamran University, Ahvaz, Iran

Tel: 0098-916-311-6165Ｅ-mail: javad1950@gmail.com

Hasan Zakidizaji

Department of Agricultural Machinery, Faculty of Agriculture

Shahid Chamran University, Ahvaz, Iran

Tel: 0098-912-479-3407Ｅ-mail: hzakid@scu.ac.ir

\begin{abstract}
Afshin Marzban
Department of Agricultural Machinery

Ramin Agriculture and Natural Resources University, Mollasani, Ahvaz, Iran
\end{abstract}

Tel: 0098-916-618-2246Ｅ-mail: afshinmarzban@hotmail.com

Mahmood Shomeili

Manager of Agronomy Department in Iranian Sugarcane Research and Training Institute

Tel: 0098-916-853-3386Ｅ-mail: eshomeli@gmail.com 
Received: April 14, 2015 Accepted: April 30, 2015

doi:10.5296/jas.v3i2.7420

URL: http://dx.doi.org/10.5296/jas.v3i2.7420

\begin{abstract}
Analysis and evaluation of agricultural systems use these criteria: energy, economic, agronomy, environmental conservation and time. Because of time importance indicator for reducing timeliness cost, project scheduling techniques are used. Graphical Evaluation and Review Technique (GERT) is widely used as a tool for managing projects. In this research GERT Networks were used and operations scheduling of sugarcane production (land preparation, planting and preserve operations) in Khuzestan province of Iran as a case study was analysed, by using WinQsb software. Critical activities, events and path were determined. The earliest project completion time is 214.03 days. The results show a high potential for operations scheduling of sugarcane production.
\end{abstract}

Keywords: Scheduling, GERT network, Agricultural Mechanization, Sugarcane

\title{
1. Introduction
}

Analysis and evaluation of agricultural systems use these criteria: energy, economic, agronomy, environmental conservation and time. Because of time importance indicator for reducing timeliness cost and work breakdown, project scheduling techniques and work study especially network models are used. Such a network would as a powerful tool available a farm manager to plan, schedule, monitor, and control a project (Monjezi et al, 2012a). Since GERT (Graphical Evaluation and Review Technique) Networks have most of the advantages associated with networks and enables system analyst in exact evaluation of certain types of networks, in this research GERT Networks were used and operations scheduling of sugarcane production in Khuzestan province of Iran as a case study was analysed. Manju and Pooja (2007) GERT technique was applied to model and analyse the reliability of the above system. One of the strengths of the GERT network is the graphical representation, which is intuitive and easy to understand (Manju \& Pooja, 2007). Abdi et al. performed Modeling and Analysis of Mechanization Projects of Wheat Production by GERT Networks. Results showed that the network model was able to answer any statistic questions concerning with the project (Abdi et al, 2010 and Abdi et al, 2009).

\section{Materials and Methods}

The study was carried out in Khuzestan province of Iran in 2015. Data were collected from variety sources such as reports and statistics of meteorological synoptic stations, opinions and comments of Khuzestan Sugarcane and by-Product Research and Training Institute experts and reports and statistics of Sugarcane Agro-Industry. All activity times are given in day. Having known perform once probability $\left(\mathrm{p}_{\mathrm{ij}}\right)$ and three time estimates for an activity: optimistic time $\left(\mathrm{t}_{\mathrm{o}}\right)$, most likely time $\left(\mathrm{t}_{\mathrm{m}}\right)$ and pessimistic time $\left(\mathrm{t}_{\mathrm{p}}\right)$, then expected time $\left(\mathrm{t}_{\mathrm{e}}\right)$ and variance $\left(\mathrm{v}_{\mathrm{te}}\right)$ was calculated for an activity from formulas (1) and (2): 


$$
\begin{gathered}
t_{e}=\mu=\frac{t_{0}+4 t_{m}+t_{p}}{6} \\
v_{t_{e}}=\left(\frac{t_{p}-t_{0}}{6}\right)^{2}
\end{gathered}
$$

The leading route calculation (the first node of the last node in the network starts to go) as soon as the expected (mean) and its variance of the occurrence of any event, relations (3) and (4) are calculated, respectively.

$$
\begin{gathered}
\mu_{T_{E}}^{j}=\max \left\{\mu_{T_{E}}^{j}+t_{e}^{i j}, \ldots\right\} \\
\delta_{T_{E}}^{2 j}=\delta_{T_{E}}^{2 i}+v_{t_{e}}^{i j}
\end{gathered}
$$

Where:

$\mu_{\mathrm{T}_{\mathrm{E}}^{\mathrm{j}}}^{\mathrm{j}}$ : Expected earliest occurrence time

$\mathrm{T}_{\mathrm{E}}$ : Earliest time of occurrence of a random variable

$\delta_{\mathrm{T}_{\mathrm{E}}}^{2 \mathrm{j}}$ : Earliest time the event variance

And in computing the backward direction (from the last node to the first node in the network has begun and will continue) latest times the expected (mean) and variance of the occurrence of any event that the relations (5) and (6) are obtained.

$$
\begin{gathered}
\mu_{\mathrm{T}_{\mathrm{L}}}^{\mathrm{i}}=\min \left\{\mu_{\mathrm{T}_{\mathrm{L}}}^{\mathrm{j}}-\mathrm{t}_{\mathrm{e}^{3}, \ldots}^{\mathrm{ij}}\right\} \\
\delta_{\mathrm{T}_{\mathrm{L}}}^{2 \mathrm{i}} \delta_{\mathrm{T}_{\mathrm{L}}}^{2 \mathrm{j}}+v_{\mathrm{t}_{e}}^{\mathrm{ij}}
\end{gathered}
$$

Where:

$\mu_{T_{L}}^{\mathrm{i}}$ : Expected latest occurrence time 
$\mathrm{T}_{\mathrm{L}}$ : Latest time of occurrence of a random variable

$\delta_{\mathrm{T}_{\mathrm{L}}}^{2 \mathrm{i}}$

: Latest time the event variance

Events in GERT slack for calculating network, since the slack for the event is the general latest time minus the earliest time of occurrence of the event and also the network and the earliest time of occurrence of GERT latest both random variables are defined, So the slack (S) is also a random variable from equation (7) is calculated.

$$
\mathrm{S}=\mathrm{T}_{\mathrm{L}}-\mathrm{T}_{\mathrm{E}}
$$

The slack is obtained by subtracting two independent random variables with a normal distribution and to calculate the mean and variance of the relations (8) and (9) are used, respectively.

$$
\begin{aligned}
& \mu_{\mathrm{S}}=\mathrm{E}(\mathrm{S})=\mu_{\mathrm{T}_{\mathrm{L}}}-\mu_{\mathrm{T}_{\mathrm{E}}} \\
& \delta_{\mathrm{S}}^{2}=\operatorname{var}(\mathrm{s})=\delta_{\mathrm{T}_{\mathrm{L}}}^{2}+\delta_{\mathrm{T}_{\mathrm{E}}}^{2}
\end{aligned}
$$

All calculations were performed using the software Win QSB (Windows Quantitative System for Business).

\section{Results and Discussion}

Time estimates (optimistic time, most likely time and pessimistic time), probability and Variance for each activity of sugarcane production (land preparation, planting and preserve operations) were calculated (Table 1). The results of Activity Analysis for project scheduling (project completion time, critical activities, earliest and latest start time, earliest and latest finish time and slack time) of sugarcane production, by using WinQsb software, have been shown in Table 1. The Table 1 shows that the earliest project completion time is 214.03 days. Some activities have a positive slack and some may have zero slack. Positive slack for each activity, showing the progress of the project ahead of schedule. In fact, there are many sources for that activity. Zero slack means being critical of the activity, the activity must occur at a specific time; otherwise they will schedule the project. Zero slack in progress, indicates the progress of the project schedule and resources are appropriately allocated. Critical activities, events (event, the result of completing one or more activities), or paths, if they delayed, will delay completion of the project. A project's critical path is understood to mean that sequence of critical activities (and critical events) which connects the project's start event to its end event cannot be delayed without delaying the project [5]. In other words, a critical path defines a chain of critical activities which connects start and end events of the directed network. The method of determining such a path includes two phases: The first phase is called the forward pass where calculations begin from the 'start' node to the 'end' 
node. The objective of this phase is computation of the earliest start time ( $\left.\mathrm{T}_{\mathrm{E}}\right)$ of all events. The second phase called the backward pass begins calculation from the 'end' node and moves to the 'start' node. The objective of this phase is computation of the latest completion time $\left(\mathrm{T}_{\mathrm{L}}\right)$ for all events. Slack times is the difference between the latest completion time and the earliest start time ( $\left.\mathrm{Si}=\mathrm{T}_{\mathrm{Li}-} \mathrm{T}_{\mathrm{Ei}}\right) . \mathrm{S}, \mathrm{T}_{\mathrm{L}}$ and $\mathrm{T}_{\mathrm{E}}$ for each event and the results of computations are presented in Table 1:

(1) $\mathrm{T}_{\mathrm{EEND}}=214.03$ (day) is the earliest completion time for event END and whole project;

(2) $\mathrm{T}_{\mathrm{LEND}}=214.03$ (day) is the latest completion time for event END and whole project (The researcher assumed that $\mathrm{T}_{\mathrm{L}}$ of project equal to $\mathrm{T}_{\mathrm{E}}$ one);

(3) $\mathrm{S}=0$ presents that this activity is critical. Project's progress is according to the scheduling and the resource allocation is proper;

(4) $\mathrm{S}=\mathrm{n}, \mathrm{n}>0$. Project progress is foregoing than scheduling and resources are surplus;

(5) $\mathrm{S}=\mathrm{m}, \mathrm{m}<0$. Project progress is lag behind than scheduling and resources are lack;

(6) Critical path, events and activities are known. The critical path is of great interest for project managers. The activities on the critical path are ones which absolutely must be done on time in order to complete the whole project on time. If any activity on the critical

(7) Given the critical path, the earliest expected time for completion of the first part of sugarcane production operations shall be to:

$$
\begin{aligned}
\mu_{T_{E}}^{118}=0+3.16 & +0.1+0.1+5+0+8.16+0+1+4+1+4+1.83+8+1+8 \\
& +1.83+8+1+7+4+1+4+1.83+4+1.83+2+1.83+1+9 \\
& +1+9+1.83+2.08+1+4.25+3+4.25+1+0+1.83+4.16+0 \\
& +4.25+1+0+2+7.16+0+4.25+1.83+4+4.25+5+1+7.17 \\
& +1+1+0+1.83+3+0+4.25+1+0+1.83+2.08+0+4.25 \\
& +1.83+1+13.83+2+0+1.83+4.25+0=214.03
\end{aligned}
$$

And also taking into consideration the critical path, the variance of the end activity will be as follows: 


$$
\begin{aligned}
\delta_{\mathrm{T}_{\mathrm{E}}^{118}}^{2^{118}}=0.250 & +0+0+0.111+0+0.250+0+0+0.111+0+0.111+0.027 \\
& +0.111+0+0.111+0.027+0.111+0+0.111+0.111+0+0.111 \\
& +0.027+0.111+0.027+0.111+0.027+0+0.111+0+0.111 \\
& +0.027+0.062+0+0.174+0.111+0.174+0+0+0.027+0.694 \\
& +0+0.174+0+0+0.111+0.694+0+0.174+0.027+0.111 \\
& +0.174+0.444+0+0694+0+0+0+0.027+0.111+0+0.174 \\
& +0+0+0.027+0.062+0+0.174+0.027+0+4.694+0+0 \\
& +0.027+0.174+0=11.41
\end{aligned}
$$

This course may be taken with respect to the probability of finding the directories in the path that is provided in Table 1, as follows:

$$
P_{\text {critical path }}=0.25 \times 0.2 \times 0.25 \times 0.25 \times 0.5 \times 0.9=0.0014
$$

\section{Conclusion}

In this research GERT Networks were used and operations scheduling of sugarcane production (land preparation, planting and preserve operations) in Khuzestan province of Iran as a case study was analysed, by using WinQsb software. Critical activities, events and path were determined. The earliest project completion time is 214.03 days. The results show a high potential for operations scheduling of sugarcane production. 


\section{Macrothink IInstitute}

Table 1. Computation results and analysis of sugarcane production classical GERT network

\begin{tabular}{|c|c|c|c|c|c|c|c|c|c|c|c|c|c|c|c|c|c|c|}
\hline \multirow{2}{*}{$\begin{array}{l}\text { Activity } \\
\text { code }\end{array}$} & \multirow[t]{2}{*}{ Activity description } & \multirow{2}{*}{$\begin{array}{l}\text { Immediate } \\
\text { predecessor }\end{array}$} & \multirow[t]{2}{*}{$t_{0}$} & \multirow[t]{2}{*}{$t_{m}$} & \multirow[t]{2}{*}{$t_{p}$} & \multirow[t]{2}{*}{$t_{e}$} & \multirow[t]{2}{*}{$v_{t_{z}}$} & \multirow[t]{2}{*}{$p_{\mathrm{i} j}$} & \multicolumn{2}{|c|}{ Start time } & \multicolumn{2}{|c|}{ Finish time } & \multicolumn{2}{|c|}{$\begin{array}{l}\text { Start time } \\
\text { variance }\end{array}$} & \multicolumn{2}{|c|}{$\begin{array}{c}\text { Finish time } \\
\text { variance }\end{array}$} & \multirow[t]{2}{*}{$\begin{array}{c}\text { Slack } \\
\text { (LS-ES) }\end{array}$} & \multirow{2}{*}{$\begin{array}{c}\text { Variance } \\
\text { slack }\end{array}$} \\
\hline & & & & & & & & & $\mathrm{ES}$ & LS & $\mathrm{EF}$ & LF & VES & VLS & VEF & VLF & & \\
\hline $\mathrm{S}$ & START & - & 0 & 0 & 0 & 0 & 0 & 1 & 0 & 0 & 0 & 0 & 0 & 0 & 0 & 0 & 0 & 0 \\
\hline 001 & Sampling of soil & $S$ & 1 & 1 & 1 & 1 & 0 & 1 & 0 & 4.36 & 1 & 5.36 & 0 & 0.25 & 0 & 0.25 & 4.36 & 0.25 \\
\hline 002 & Test results of soil & 001 & 2 & 3 & 4 & 3 & 0.111 & 1 & 1 & 5.36 & 4 & 8.36 & 0 & 0.25 & 0.11 & 0.36 & 4.36 & 0.25 \\
\hline 004 & Tractors, border and grader supply & $\mathrm{S}$ & 1 & 2 & 2 & 1.83 & 0.027 & 1 & 0 & 1.53 & 1.83 & 3.36 & 0 & 0.22 & 0.03 & 0.25 & 1.53 & 0.22 \\
\hline 005 & Operators employ & $\mathrm{S}$ & 1 & 2 & 3 & 2 & 0.111 & 1 & 0 & 1.36 & 2 & 3.36 & 0 & 0.14 & 0.11 & 0.25 & 1.36 & 0.14 \\
\hline 006 & Oil and fuel supply & $\mathrm{S}$ & 2 & 3 & 5 & 3.16 & 0.250 & 1 & 0 & 0 & 3.16 & 3.16 & 0 & 0 & 0.25 & 0.25 & 0 & 0 \\
\hline 007 & Oil and fuel for land preparation & 006 & 0.1 & 10.1 & 0.1 & 0.1 & 0 & 1 & 3.16 & 3.16 & 3.26 & 3.26 & 0.25 & 0.25 & 0.25 & 0.25 & 0 & 0 \\
\hline 008 & Oil and fuel for bordering & 007 & 0.1 & 10.1 & 0.1 & 0.1 & 0 & 1 & 3.26 & 3.26 & 3.36 & 3.36 & 0.25 & 0.25 & 0.25 & 0.25 & 0 & 0 \\
\hline 010 & Decide to leaching & 002,009 & 0 & 0 & 0 & 0 & 0 & 0.25 & 8.36 & 8.36 & 8.36 & 8.36 & 0.36 & 0.36 & 0.36 & 0.36 & 0 & 0 \\
\hline 011 & Decide to non- leaching & 002,009 & 0 & 0 & 0 & 0 & 0 & 0.75 & 8.36 & 11.53 & 8.36 & 11.53 & 0.36 & 0.5 & 0.36 & 0.5 & 3.16 & 0.14 \\
\hline 012 & Leaching & 010 & 7 & 8 & 10 & 8.16 & 0.250 & 1 & 8.36 & 8.36 & 16.53 & 16.53 & 0.36 & 0.36 & 0.61 & 0.61 & 0 & 0 \\
\hline 013 & Pre-irrigation & 011 & 4 & 5 & 6 & 5 & 0.111 & 1 & 8.36 & 11.53 & 13.36 & 16.53 & 0.36 & 0.5 & 0.47 & 0.61 & 3.16 & 0.14 \\
\hline 014 & Decide to disc harrowing & 012 & 0 & 0 & 0 & 0 & 0 & 1 & 16.53 & 16.53 & 16.53 & 16.53 & 0.61 & 0.61 & 0.61 & 0.61 & 0 & 0 \\
\hline 015 & Decide to non- disc harrowing & 013 & 0 & 0 & 0 & 0 & 0 & 1 & 13.36 & 16.53 & 13.36 & 16.53 & 0.47 & 0.61 & 0.47 & 0.61 & 3.16 & 0.14 \\
\hline 016 & Disc harrow supply & 014,015 & 1 & 1 & 1 & 1 & 0 & 1 & 16.53 & 16.53 & 17.53 & 17.53 & 0.61 & 0.61 & 0.61 & 0.61 & 0 & 0 \\
\hline 017 & Oil and fuel for disc harrowing & 007 & 0.1 & 10.1 & 0.1 & 0.1 & 0 & 1 & 3.26 & 17.43 & 3.36 & 17.53 & 0.25 & 0.61 & 0.25 & 0.61 & 14.16 & 0.36 \\
\hline 018 & Primary disc harrowing & 016,017 & 3 & 4 & 5 & 4 & 0.111 & 1 & 17.53 & 17.53 & 21.53 & 21.53 & 0.61 & 0.61 & 0.72 & 0.72 & 0 & 0 \\
\hline 019 & Leveler provide & 018 & 1 & 1 & 1 & 1 & 0 & 1 & 21.53 & 21.53 & 22.53 & 22.53 & 0.72 & 0.72 & 0.72 & 0.72 & 0 & 0 \\
\hline
\end{tabular}




\section{Macrothink}

Journal Title XXXXXXX

ISSN XXXX-XXXX

201X, Vol. X, No. X

\begin{tabular}{|c|c|c|c|c|c|c|c|c|c|c|c|c|c|c|c|c|c|c|}
\hline \multirow{2}{*}{$\begin{array}{l}\text { Activity } \\
\text { code }\end{array}$} & \multirow{2}{*}{ Activity description } & \multirow{2}{*}{$\begin{array}{l}\text { Immediate } \\
\text { predecessor }\end{array}$} & \multirow[t]{2}{*}{$t_{0} t$} & \multirow[t]{2}{*}{$\left.t_{\mathrm{m}}\right|^{t}$} & \multirow[t]{2}{*}{$t_{p}$} & \multirow[t]{2}{*}{$t_{e}$} & \multirow[t]{2}{*}{$v_{t_{s}}$} & \multirow[t]{2}{*}{$p_{\mathrm{i} j}$} & \multicolumn{2}{|c|}{ Start time } & \multicolumn{2}{|c|}{ Finish time } & \multicolumn{2}{|c|}{$\begin{array}{l}\text { Start time } \\
\text { variance }\end{array}$} & \multicolumn{2}{|c|}{$\begin{array}{l}\text { Finish time } \\
\text { variance }\end{array}$} & \multirow[t]{2}{*}{$\begin{array}{c}\text { Slack } \\
(\mathrm{LS}-\mathrm{ES})\end{array}$} & \multirow{2}{*}{$\begin{array}{l}\text { Variance } \\
\text { slack }\end{array}$} \\
\hline & & & & & & & & & ES & LS & $\mathrm{EF}$ & $\mathrm{LF}$ & VES & VLS & VEF & VLF & & \\
\hline 021 & Primary leveling & 019,020 & 3 & 4 & 5 & 4 & 0.111 & 1 & 22.53 & 22.53 & 26.53 & 26.53 & 0.72 & 0.72 & 0.83 & 0.83 & 0 & 0 \\
\hline 022 & Scraper provide & 021 & 1 & 2 & 2 & 1.83 & 0.027 & 1 & 26.53 & 26.53 & 28.36 & 28.36 & 0.83 & 0.83 & 0.86 & 0.86 & 0 & 0 \\
\hline 024 & Scrapering & 022,023 & 7 & 8 & 9 & 8 & 0.111 & 1 & 28.36 & 28.36 & 36.36 & 36.36 & 0.86 & 0.86 & 0.97 & 0.97 & 0 & 0 \\
\hline 025 & Mold board plow provide & 024 & 1 & 1 & 1 & 1 & 0 & 1 & 36.36 & 36.36 & 37.36 & 37.36 & 0.97 & 0.97 & 0.97 & 0.97 & 0 & 0 \\
\hline 026 & Oil and fuel for plowing & 007 & $0.1 \mathrm{C}$ & 0.10 & 0.1 & 0.1 & 0 & 1 & 3.26 & 37.26 & 3.36 & 37.36 & 0.25 & 0.97 & 0.25 & 0.97 & 34 & 0.72 \\
\hline 027 & Plowing & 025,026 & 7 & 8 & 9 & 8 & 0.111 & 1 & 37.36 & 37.36 & 45.36 & 45.36 & 0.97 & 0.97 & 1.08 & 1.08 & 0 & 0 \\
\hline 028 & Bulldozer and subsoiler supply & 027 & 1 & 2 & 2 & 1.83 & 0.027 & 1 & 45.36 & 45.36 & 47.2 & 47.2 & 1.08 & 1.08 & 1.11 & 1.11 & 0 & 0 \\
\hline 030 & Subsoiling & 028,029 & 7 & 8 & 9 & 8 & 0.111 & 1 & 47.2 & 47.2 & 55.2 & 55.2 & 1.11 & 1.11 & 1.22 & 1.22 & 0 & 0 \\
\hline 031 & Tractors and disc harrow supply & 030 & 1 & 1 & 1 & 1 & 0 & 1 & 55.2 & 55.2 & 56.2 & 56.2 & 1.22 & 1.22 & 1.22 & 1.22 & 0 & 0 \\
\hline 032 & Oil and fuel for second disc harrowing & 007 & 0.1 & 0.10 & 0.1 & 0.1 & 0 & 1 & 3.26 & 56.1 & 3.36 & 56.2 & 0.25 & 1.22 & 0.25 & 1.22 & 52.83 & 0.97 \\
\hline 033 & Second disc harrowing & 031,032 & 6 & 7 & 8 & 7 & 0.111 & 1 & 56.2 & 56.2 & 63.2 & 63.2 & 1.22 & 1.22 & 1.33 & 1.33 & 0 & 0 \\
\hline 034 & Second leveling & 033 & 3 & 4 & 5 & 4 & 0.111 & 1 & 63.2 & 63.2 & 67.2 & 67.2 & 1.33 & 1.33 & 1.44 & 1.44 & 0 & 0 \\
\hline 035 & Furrower supply & 034 & 1 & 1 & 1 & 1 & 0 & 1 & 67.2 & 67.2 & 68.2 & 68.2 & 1.44 & 1.44 & 1.44 & 1.44 & 0 & 0 \\
\hline 036 & Oil and fuel for furrowing & 007 & 0.1 & 0.10 & 0.1 & 0.1 & 0 & 1 & 3.26 & 68.1 & 3.36 & 68.2 & 0.25 & 1.44 & 0.25 & 1.44 & 64.83 & 1.19 \\
\hline 037 & Furrowing & 035,036 & 3 & 4 & 5 & 4 & 0.111 & 1 & 68.2 & 68.2 & 72.2 & 72.2 & 1.44 & 1.44 & 1.55 & 1.55 & 0 & 0 \\
\hline 038 & $\begin{array}{l}\text { Chemical fertilizer and fertilizer attachments } \\
\text { supply }\end{array}$ & 037 & 1 & 2 & 2 & 1.83 & 0.027 & 1 & 72.2 & 72.2 & 74.03 & 74.03 & 1.55 & 1.55 & 1.58 & 1.58 & 0 & 0 \\
\hline 039 & Oil and fuel for fertilizering & 007 & 0.1 & 0.10 & 0.1 & 0.1 & 0 & 1 & 3.26 & 73.93 & 3.36 & 74.03 & 0.25 & 1.58 & 0.25 & 1.58 & 70.66 & 1.33 \\
\hline
\end{tabular}




\section{Macrothink \\ institute ${ }^{\text {TM }}$}

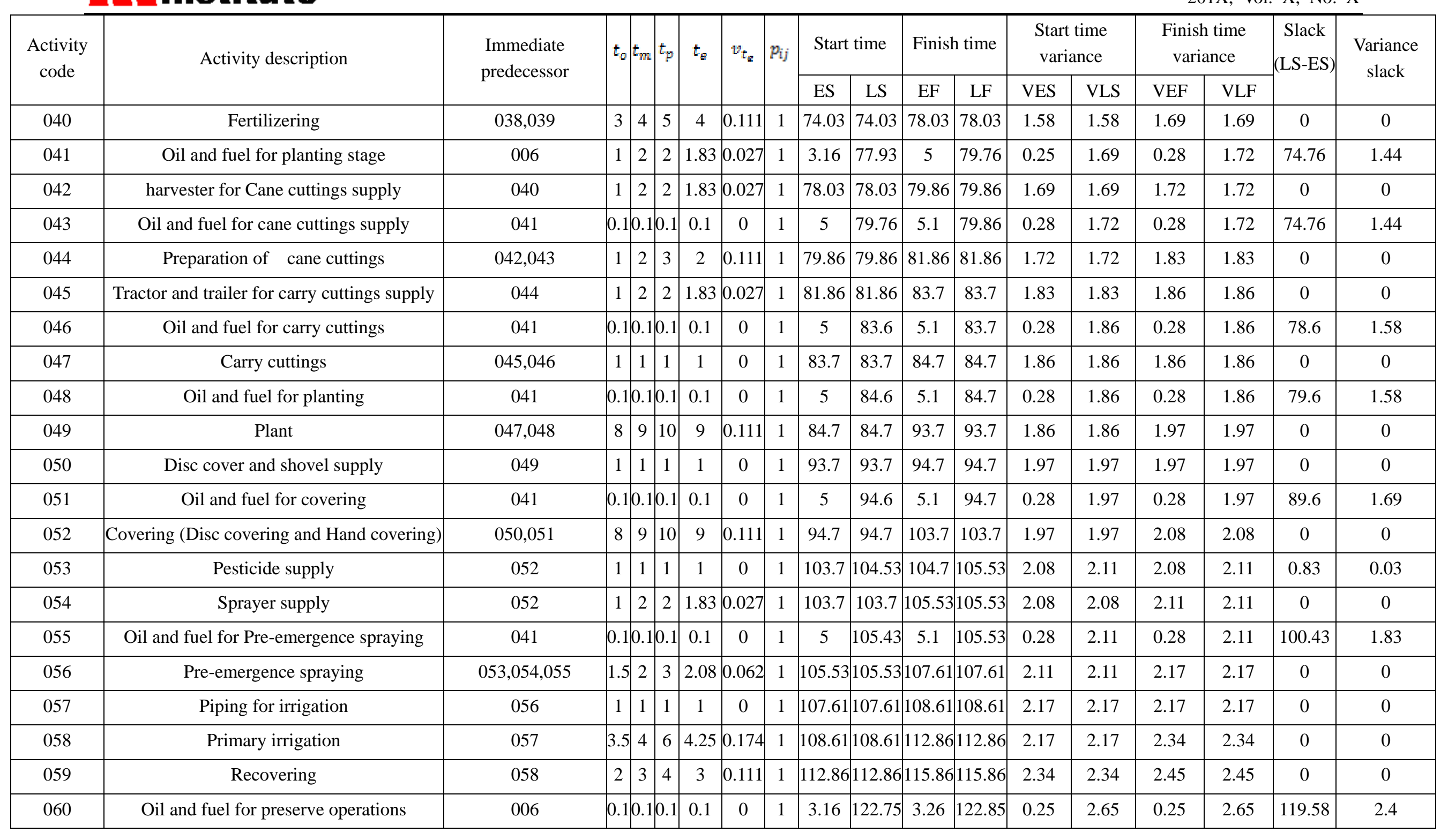




\section{Macrothink}

Journal Title XXXXXXX

ISSN XXXX-XXXX

201X, Vol. X, No. X

\begin{tabular}{|c|c|c|c|c|c|c|c|c|c|c|c|c|c|c|c|c|c|c|}
\hline \multirow{2}{*}{$\begin{array}{l}\text { Activity } \\
\text { code }\end{array}$} & \multirow[t]{2}{*}{ Activity description } & \multirow{2}{*}{$\begin{array}{l}\text { Immediate } \\
\text { predecessor }\end{array}$} & \multirow[t]{2}{*}{$t_{0} t_{3}$} & \multirow{2}{*}{\multicolumn{2}{|c|}{$t_{p}$}} & \multirow[t]{2}{*}{$t_{\text {e }}$} & \multirow[t]{2}{*}{$v_{t_{s}}$} & \multirow[t]{2}{*}{$p_{i j}$} & \multicolumn{2}{|c|}{ Start time } & \multicolumn{2}{|c|}{ Finish time } & \multicolumn{2}{|c|}{$\begin{array}{l}\text { Start time } \\
\text { variance }\end{array}$} & \multicolumn{2}{|c|}{$\begin{array}{l}\text { Finish time } \\
\text { variance }\end{array}$} & \multirow[t]{2}{*}{$\begin{array}{c}\text { Slack } \\
(\mathrm{LS}-\mathrm{ES})\end{array}$} & \multirow{2}{*}{$\begin{array}{l}\text { Variance } \\
\text { slack }\end{array}$} \\
\hline & & & & & & & & & ES & LS & $\mathrm{EF}$ & $\mathrm{LF}$ & VES & VLS & VEF & VLF & & \\
\hline 062 & Visit the farm (Evaluation of green field) & 061 & 1 & 1 & 1 & 1 & 0 & 1 & 120.11 & $120.11 \mid 1$ & 121.11 & 121.11 & 2.62 & 2.62 & 2.62 & 2.62 & 0 & 0 \\
\hline 063 & Decide to non- replant & 062 & 0 & 0 & 0 & 0 & 0 & 0.8 & 121.11 & $127.11 \mid 1$ & 121.11 & 127.11 & 2.62 & 3.34 & 2.62 & 3.34 & 6 & 0.72 \\
\hline 065 & Oil and fuel for replant & 060 & 0.10 & 0.10 & 0.1 & 0.1 & 0 & 1 & 3.261 & 122.85 & 3.36 & 122.95 & 0.25 & 2.65 & 0.25 & 2.65 & 119.58 & 2.4 \\
\hline 066 & $\begin{array}{l}\text { Tractors, trailer, cane cutter and shovel } \\
\text { supply }\end{array}$ & 064 & 1 & 2 & 21 & 1.83 & 0.027 & 1 & 121.11 & $121.11 \mid 1$ & 122.95 & 122.95 & 2.62 & 2.62 & 2.65 & 2.65 & 0 & 0 \\
\hline 067 & Preparation of cane cuttings & 064 & 1 & 1 & 1 & 1 & 0 & 1 & 121.11 & 121.951 & 122.11 & 122.95 & 2.62 & 2.65 & 2.62 & 2.65 & 0.83 & 0.03 \\
\hline 068 & Replant & $065,066,067$ & 2 & 4 & $7 \mid 4$ & 4.16 & 0.694 & 1 & 122.95 & 122.951 & 127.11 & 127.11 & 2.65 & 2.65 & 3.34 & 3.34 & 0 & 0 \\
\hline 070 & Decide to irrigation & 063 & 0 & 0 & 0 & 0 & 0 & 1 & 121.11 & 127.111 & 121.11 & 127.11 & 2.62 & 3.34 & 2.62 & 3.34 & 6 & 0.72 \\
\hline 071 & Irrigation & 069,070 & 3.5 & 4 & 64 & 4.25 & 0.174 & 1 & $|127.11|$ & 127.111 & 131.36 & 131.36 & 3.34 & 3.34 & 3.51 & 3.51 & 0 & 0 \\
\hline 072 & Visit the farm (weed infestation) & 071 & 1 & 1 & 1 & 1 & 0 & 1 & 131.36 & 131.361 & 132.36 & 132.36 & 3.51 & 3.51 & 3.51 & 3.51 & 0 & 0 \\
\hline 073 & Decide to non- mechanical weed control & 072 & 0 & 0 & 0 & 0 & 0 & 0.75 & 132.36 & 141.531 & 132.36 & 141.53 & 3.51 & 4.31 & 3.51 & 4.31 & 9.16 & 0.8 \\
\hline 074 & Decide to mechanical weed control & 072 & 0 & 0 & 0 & 0 & 0 & 0.25 & 132.36 & 132.361 & 132.36 & 132.36 & 3.51 & 3.51 & 3.51 & 3.51 & 0 & 0 \\
\hline 075 & Labor, shovel and sickle supply & 074 & 1 & 2 & 3 & 2 & 0.111 & 1 & $|132.36|$ & 132.361 & 134.36 & 134.36 & 3.51 & 3.51 & 3.62 & 3.62 & 0 & 0 \\
\hline 076 & mechanical weed control & 075 & 5 & 7 & 107 & 7.16 & 0.694 & 1 & $134.36 \mid$ & 134.36 & 141.53 & 141.53 & 3.62 & 3.62 & 4.31 & 4.31 & 0 & 0 \\
\hline 077 & Decide to irrigation & 076 & 0 & 0 & 0 & 0 & 0 & 1 & 141.53 & 141.531 & 141.53 & 141.53 & 4.31 & 4.31 & 4.31 & 4.31 & 0 & 0 \\
\hline 078 & Decide to irrigation & 073 & 0 & 0 & 0 & 0 & 0 & 1 & 132.36 & 141.531 & 132.36 & 141.53 & 3.51 & 4.31 & 3.51 & 4.31 & 9.16 & 0.8 \\
\hline 079 & Irrigation & 077,078 & 3.5 & 4 & 65 & 4.25 & 0.174 & 1 & 141.53 & 141.531 & 145.78 & 145.78 & 4.31 & 4.31 & 4.48 & 4.48 & 0 & 0 \\
\hline 080 & Oil and fuel for hilling up & 060 & 0.10 & 0.10 & 0.1 & 0.1 & 0 & 1 & 3.261 & 147.51 & 3.36 & 147.61 & 0.25 & 4.51 & 0.25 & 4.51 & 144.25 & 4.26 \\
\hline
\end{tabular}




\section{Macrothink \\ institute ${ }^{\text {TM }}$}

\begin{tabular}{|c|c|c|c|c|c|c|c|c|c|c|c|c|c|c|c|c|c|c|}
\hline \multirow{2}{*}{$\begin{array}{l}\text { Activity } \\
\text { code }\end{array}$} & \multirow[t]{2}{*}{ Activity description } & \multirow{2}{*}{$\begin{array}{l}\text { Immediate } \\
\text { predecessor }\end{array}$} & \multirow[t]{2}{*}{$t_{0}$} & \multirow[t]{2}{*}{$m$} & $t_{p}$ & \multirow[t]{2}{*}{$t_{e}$} & \multirow[t]{2}{*}{$v_{t_{z}}$} & \multirow[t]{2}{*}{$p_{i j}$} & \multicolumn{2}{|c|}{ Start time } & \multicolumn{2}{|c|}{ Finish time } & \multicolumn{2}{|c|}{$\begin{array}{l}\text { Start time } \\
\text { variance }\end{array}$} & \multicolumn{2}{|c|}{$\begin{array}{l}\text { Finish time } \\
\text { variance }\end{array}$} & \multirow[t]{2}{*}{$\begin{array}{c}\text { Slack } \\
(\mathrm{LS}-\mathrm{ES})\end{array}$} & \multirow{2}{*}{$\begin{array}{l}\text { Variance } \\
\text { slack }\end{array}$} \\
\hline & & & & & & & & & ES & $\mathrm{LS}$ & $\mathrm{EF}$ & $\mathrm{LF}$ & VES & VLS & VEF & VLF & & \\
\hline 081 & Hilling up implement supply & 079 & 1 & 2 & 2 & 1.83 & 0.027 & 1 & 145.78 & 145.78 & 147.61 & 147.61 & 4.48 & 4.48 & 4.51 & 4.51 & 0 & 0 \\
\hline 082 & Hilling up & 080,081 & 3 & 4 & 5 & 4 & 0.111 & 1 & 147.61 & 147.61 & 151.61 & 151.61 & 4.51 & 4.51 & 4.62 & 4.62 & 0 & 0 \\
\hline 083 & Irrigation & 082 & 3.5 & 4 & 6 & 4.25 & 0.174 & 1 & 151.61 & $151.61 \mid 1$ & 155.86 & 155.86 & 4.62 & 4.62 & 4.79 & 4.79 & 0 & 0 \\
\hline 084 & Optical trap provide & 083 & 3 & 5 & 7 & 5 & 0.444 & 1 & 155.86 & 155.86 & 160.86 & 160.86 & 4.79 & 4.79 & 5.23 & 5.23 & 0 & 0 \\
\hline 085 & Mechanical pest control (optical trap) & 084 & 1 & 1 & 1 & 1 & 0 & 1 & 160.86 & 160.86 & 161.86 & 161.86 & 5.23 & 5.23 & 5.23 & 5.23 & 0 & 0 \\
\hline 086 & Parasitoid wasps supply & 085 & 5 & 7 & 10 & 7.17 & 0.694 & 1 & 161.86 & 161.86 & 169.03 & 169.03 & 5.23 & 5.23 & 5.92 & 5.92 & 0 & 0 \\
\hline 087 & $\begin{array}{l}\text { Biological pest control- parasitoid wasps } \\
\text { (first stage) }\end{array}$ & 086 & 1 & 1 & 1 & 1 & 0 & 1 & 169.03 & 169.03 & 170.03 & 170.03 & 5.92 & 5.92 & 5.92 & 5.92 & 0 & 0 \\
\hline 088 & Visit the farm (weed infestation) & 087 & 1 & 1 & 1 & 1 & 0 & 1 & 170.03 & 170.03 & 171.03 & 171.03 & 5.92 & 5.92 & 5.92 & 5.92 & 0 & 0 \\
\hline 090 & $\begin{array}{l}\text { Decide to mechanical weed control } \\
\text { (cultivator) }\end{array}$ & 088 & 0 & 0 & 0 & 0 & 0 & 0.25 & 171.03 & 171.03 & 171.03 & 171.03 & 5.92 & 5.92 & 5.92 & 5.92 & 0 & 0 \\
\hline 091 & Oil and fuel for cultivator & 060 & 0.1 & 0.1 & 0.1 & 0.1 & 0 & 1 & 3.26 & 172.86 & 3.36 & 172.86 & 0.25 & 5.95 & 0.25 & 5.95 & 169.5 & 5.7 \\
\hline 092 & Tractors and cultivator supply & 090 & 1 & 2 & 2 & 1.83 & 0.027 & 1 & 171.03 & 171.03 & 172.86 & 172.86 & 5.92 & 5.92 & 5.95 & 5.95 & 0 & 0 \\
\hline 093 & mechanical weed control (cultivator) & 091,092 & 2 & 3 & 4 & 3 & 0.111 & 1 & 172.86 & 172.86 & 175.86 & 175.86 & 5.95 & 5.95 & 6.06 & 6.06 & 0 & 0 \\
\hline 094 & Decide to irrigation & 093 & 0 & 0 & 0 & 0 & 0 & 1 & 175.86 & 175.86 & 175.86 & 175.86 & 6.06 & 6.06 & 6.06 & 6.06 & 0 & 0 \\
\hline 095 & Decide to irrigation & 089 & 0 & 0 & 0 & 0 & 0 & 1 & 171.03 & 175.86 & 171.03 & 175.86 & 5.92 & 6.06 & 5.92 & 6.06 & 4.83 & 0.14 \\
\hline 096 & Irrigation & 094,095 & 3.5 & 4 & 6 & 4.25 & 0.174 & 1 & 175.86 & 175.86 & 180.11 & 180.11 & 6.06 & 6.06 & 6.23 & 6.23 & 0 & 0 \\
\hline 097 & Visit the farm (weed infestation) & 096 & 1 & 1 & 1 & 1 & 0 & 1 & 180.11 & 180.11 & 181.11 & 181.11 & 6.23 & 6.23 & 6.23 & 6.23 & 0 & 0 \\
\hline 098 & Decide to non- herbicide spraying & 097 & 0 & 0 & 0 & 0 & 0 & 0.5 & 181.11 & 185.03 & 181.11 & 185.03 & 6.23 & 6.32 & 6.23 & 6.32 & 3.91 & 0.09 \\
\hline
\end{tabular}




\section{Macrothink}

Journal Title XXXXXXX

ISSN XXXX-XXXX

201X, Vol. X, No. X

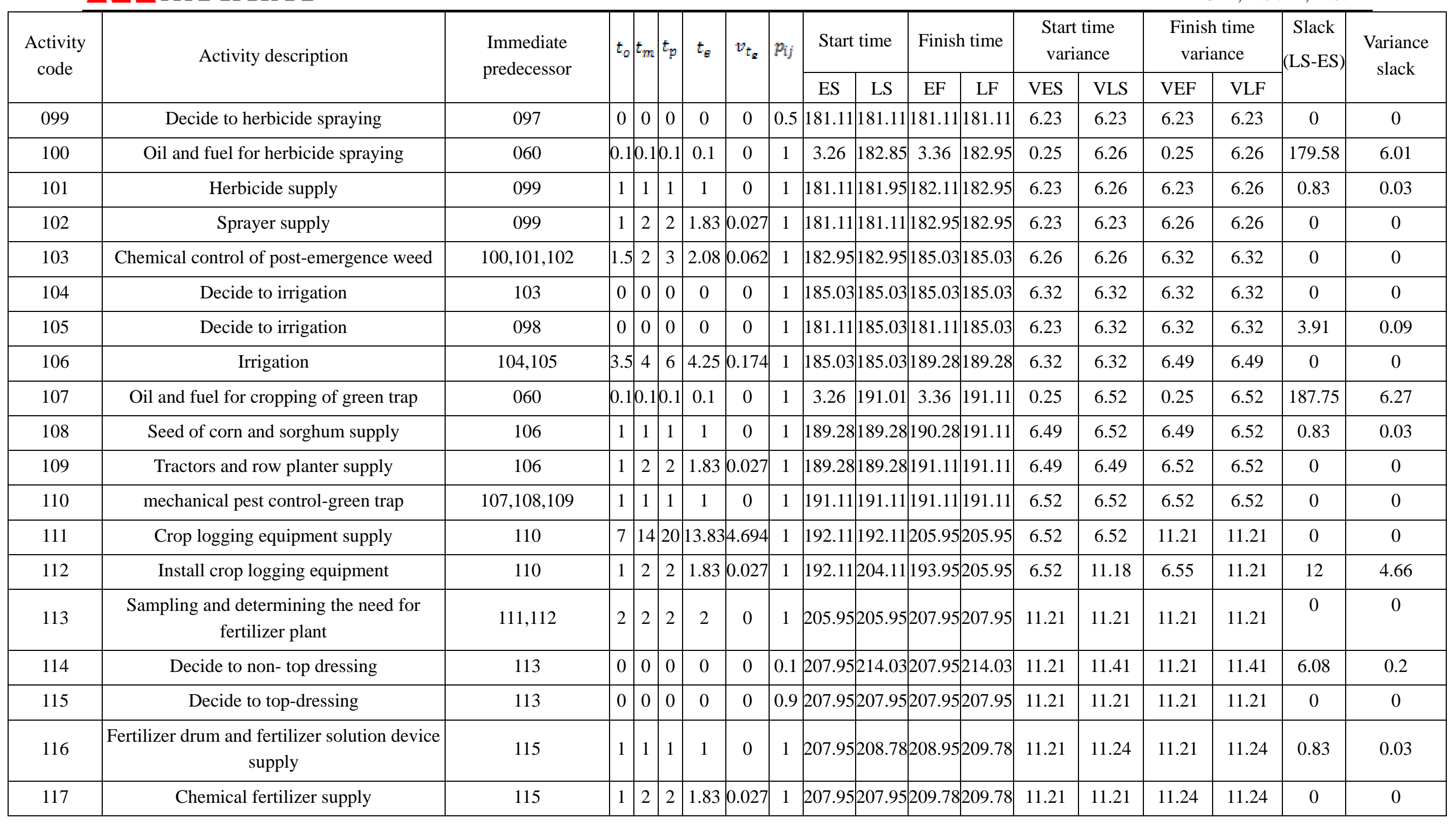




\section{Macrothink \\ Institute $^{\text {mM }}$}

\begin{tabular}{|c|c|c|c|c|c|c|c|c|c|c|c|c|c|c|c|c|c|c|}
\hline \multirow{2}{*}{$\begin{array}{l}\text { Activity } \\
\text { code }\end{array}$} & \multirow[t]{2}{*}{ Activity description } & \multirow{2}{*}{$\begin{array}{l}\text { Immediate } \\
\text { predecessor }\end{array}$} & \multirow{2}{*}{\multicolumn{2}{|c|}{$t_{0} \mid t_{3}$}} & \multirow[t]{2}{*}{$t_{p}$} & \multirow[t]{2}{*}{$t_{\text {e }}$} & \multirow[t]{2}{*}{$v_{t_{p}}$} & \multirow[t]{2}{*}{$p_{i j}$} & \multicolumn{2}{|c|}{ Start time } & \multicolumn{2}{|c|}{ Finish time } & \multicolumn{2}{|c|}{$\begin{array}{l}\text { Start time } \\
\text { variance }\end{array}$} & \multicolumn{2}{|c|}{$\begin{array}{l}\text { Finish time } \\
\text { variance }\end{array}$} & \multirow[t]{2}{*}{$\begin{array}{c}\text { Slack } \\
(\mathrm{LS}-\mathrm{ES})\end{array}$} & \multirow{2}{*}{$\begin{array}{c}\text { Variance } \\
\text { slack }\end{array}$} \\
\hline & & & & & & & & & ES & LS & $\mathrm{EF}$ & $\mathrm{LF}$ & VES & VLS & VEF & VLF & & \\
\hline $\mathrm{E}$ & END (first part of operation) & 114,118 & 0 & 0 & 0 & 0 & 0 & 1 & 214.03 & 214.03 & 214.03 & 214.03 & 11.41 & 11.41 & 11.41 & 11.41 & 0 & 0 \\
\hline
\end{tabular}




\section{References}

Abdi, R., Ghasemzadeh, H. R., Abdollahpur, S., Sabzehparvar, M., \& Mohammadi Nasab, A. D. (2009). Modeling and resource allocation of agricultural mechanization projects with GERT networks. Journal of Food, Agriculture \& Environment, 7(3\&4), 438-441.

Abdi, R., Ghasemzadeh, H. R., Abdollahpour, S., Sabzeparvar, M., \& Dabbag Mohamadi Nasab, A. (2010). Modeling and analysis of mechanization projects of wheat production by GERT networks. Elsevier, Agricultural Sciences in China, 9(7), 1078-1083. http://dx.doi.org/10.1016/S1671-2927(09)60193-0

Manju, A., \& Pooja, M. (2007). Reliability analysis of consecutive-k, r-out-of-rr. DFM system using GERT. International Journal of Operations Research, 4(2), 110-117.

Monjezi, N., Sheikhdavoodi, M. J. and Basirzadeh, H. (2012). Application of Project Scheduling in Agriculture (Case Study: Mechanized Greenhouses Construction Project). Research Journal of Applied Sciences, Engineering and Technology, 4(3), 241-244.

Monjezi, N., Sheikhdavoodi, M. J., Basirzadeh, H., \& Zakidizaji, H. (2012). Analysis and Evaluation of Mechanized Greenhouse Construction Project using CPM Methods. Research Journal of Applied Sciences, Engineering and Technology, 4(18), 3267-3273.

\section{Copyright Disclaimer}

Copyright for this article is retained by the author(s), with first publication rights granted to the journal.

This is an open-access article distributed under the terms and conditions of the Creative Commons Attribution license (http://creativecommons.org/licenses/by/3.0/). 